\title{
A low-cost phantom for ultrasound-guided thyroid fine-needle aspiration training: face and construct validity
}

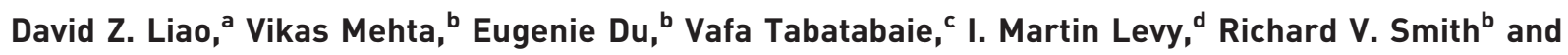 \\ Marc J. Gibber ${ }^{\mathrm{b}, *}$
}

${ }^{a}$ Albert Einstein College of Medicine, Bronx, NY, USA; ${ }^{b}$ Department of Otorhinolaryngology-Head and Neck Surgery, Montefiore Medical Center, Bronx, NY, USA; ${ }^{c}$ Division of Endocrinology, Montefiore Medical Center, Bronx, NY, USA; ${ }^{d}$ Department of Orthopedic Surgery, Montefiore Medical Center, Bronx, NY, USA

${ }^{*}$ Corresponding author at: 3400 Bainbridge Avenue, 3rd Floor MAP Building, Room 3303, Bronx, NY 10467, USA. Email: mgibber@montefiore.org

Date accepted for publication: 12 July 2020

\section{Abstract}

Background: There are currently no formally validated training phantoms for teaching ultrasound-guided thyroid fineneedle aspiration (FNA). We designed and established face and construct validity of a low-cost phantom for ultrasoundguided thyroid fine-needle aspiration. Methods: We designed an affordable, gelatin-based thyroid FNA phantom. Participants included 9 medical students, 7 novice residents/fellows ( $<5$ thyroid FNAs), and 7 expert residents/fellows $(\geqslant 5$ thyroid FNAs). Participants performed 6 trials of thyroid FNA, which were scored on completion time, accuracy, and errors. Participants also completed questionnaires to assess the benefit and realism of the simulation. Results: Simulation scores at trial 1 significantly discriminated expert participants from novice participants $(P=0.028)$ and from medical students $(P<0.001)$. Median novice resident/fellow participant scores increased with repeated trials and were no longer significantly different from expert scores by trial 2 . The scores for this group were significantly better compared with medical student scores by trial 4 . Between trial 1 and trial 6 , significant score improvements were observed in both medical students $(P=0.018)$ and novice residents $(P=0.018)$. All participants found the phantom to be beneficial in learning thyroid FNA. Both attending endocrinologists and 6 of 7 expert participants found the ultrasound visualization of the phantom to be realistic. One of two attending endocrinologists and 5 of 7 expert participants found the haptic feedback to be realistic. Conclusion: We designed a low-cost thyroid FNA phantom that demonstrates face and construct validity. This phantom can be used to train procedural skills in a residency program and can also be used to evaluate skill acquisition.

Keywords: thyroid fine-needle aspiration; ultrasound; thyroid nodule; simulation phantom; low cost

\section{Introduction}

The American Thyroid Association recommends using ultrasound-guided fine-needle aspiration (FNA) to assess thyroid nodules that are larger than $1-1.5 \mathrm{~cm}$ or that have suspicious ultrasound features. ${ }^{1}$ Practice and repetition are required for developing the three-dimensional, spatial awareness and bimanual dexterity skills required for learning thyroid FNA. FNA experience correlates with increased diagnostic rates; accuracy rates of nodule targeting in ultrasound-guided FNA are higher when performed by experienced staff. ${ }^{2}$ In addition, an improved faculty for performing thyroid FNA will lead to a decreased number of necessary passes for adequate yield and improved patient comfort. There are also many vital structures located close to the thyroid, such as the carotid artery and trachea, that may be inadvertently punctured by the needle, which increases the complexity of the procedure.

Residents can be trained in ultrasound-guided thyroid FNA using various phantoms as training models. The American Thyroid Society has historically taught ultrasound-guided thyroid FNA using turkey breasts, which carry risk of food-borne disease. ${ }^{3}$ The Blue Phantom is a high-fidelity ultrasound phantom on the market used to teach thyroid FNA but is expensive (US\$1454.96). Multiple studies have

This work has been presented at Simulation Reception and Showcase, 2019 American Academy of Otolaryngology — Head and Neck Surgery Annual Meeting and OTO Experience in New Orleans, LA, USA, September 16, 2019. 
also proposed the use of more accessible homemade ultrasound phantoms composed of agar, gelatin, silicone, Spam, chicken breast, tofu, or paraffin as tissue media and beans, olives, and berries as nodules. ${ }^{4-11}$ However, these low-cost thyroid FNA phantoms have not been formally validated.

The aim of this study was to design a low-cost, high-fidelity phantom for ultrasound-guided thyroid FNA that can be used for resident training and skill evaluation. This phantom simulate nodule targeting and is validated in two steps: (1) proving face validity based on users' subjective assessment of the realism and benefit of the phantom and (2) demonstrating discriminative construct validity by showing that users with different levels of experience will perform differently on the phantom.

\section{Materials and methods}

\section{Phantom design}

The design of the phantom incorporated inexpensive, easily obtainable materials in order to replicate the head, neck, and torso of a patient (Fig. 1A). The head was represented by any container with the dimensions of a head, and the torso was simulated by a cardboard box with shoulder pads placed on top. Within the neck, soft tissue was simulated with $12 \%$ beef gelatin, the trachea was represented with wire loom, the thyroid was created with Spam (a canned cook pork), nodules were simulated with peas (hypoechoic) and blueberries (hyperechoic), and vessels were generated by filling balloons with food coloring that bled into the phantom when punctured. A piece of latex wrap was draped over the neck phantom to simulate the elastic rebound of skin and to conceal the structures within the neck.

To fabricate the neck phantom (Fig. 1B), a mold was created with a $0.5 \mathrm{~L}$ cylindrical container. A cylindrical piece of wire loom $(6 \mathrm{~cm})$ was cut to represent the trachea. A piece of Spam was then cut into the shape of a thyroid with 1.5 $\mathrm{cm}$ thickness, $5 \mathrm{~cm}$ width, and $2.5 \mathrm{~cm}$ height with a curvature to accommodate the curvature of the wire loom. The thyroid-shaped piece of Spam was then secured to the trachea at the appropriate height by skewering a toothpick through the thyroid and wire loom. Blueberries and peas (selected to be around $1 \mathrm{~cm}$ in diameter) representing nodules could be embedded into the Spam thyroid after using a toothpick to etch out spaces. Six nodules were placed throughout the thyroid so that there would be two nodules in each of the hemispheres and the isthmus. Nodules were positioned at a consistent depth (about 1 $\mathrm{cm}$ ) from the surface of the container. Two tied long balloons representing vessels were filled with food coloring. The trachea-thyroid-nodules complex was secured to the bottom of a $0.5-\mathrm{L}$ plastic container with balloons that were secured inferolaterally on each side of the thyroid. $12 \%$ gelatin (5 tablespoons of beef gelatin stirred with 2 cups of boiling water) was poured into the mold and allowed to set overnight.

To assemble the phantom before use, the neck phantom was removed from the $0.5-\mathrm{L}$ container and placed on a carboard box simulating the dimensions of a human torso. A piece of latex wrap was added onto the neck with ultrasound gel at the interface. A pair of shoulder pads was placed on the carboard box. A container simulating a head was placed onto the neck phantom and a rigid structure (i.e. boxes of gloves) was placed behind the neck phantom for support.

\section{Face and construct validity}

Medical students (no experience with thyroid FNA), novice otolaryngology residents/endocrine fellows $(<5$ thyroid FNAs performed on live patients), and expert otolaryngology residents/endocrine fellows ( $\geqslant 5$ thyroid FNAs performed on live patients) were recruited from Albert Einstein College of Medicine/Montefiore Medical Center after the institutional review board approved this study (study number 2018-8549). The amount of previous thyroid FNA experience was determined by survey and participants were contacted by email. The threshold (5 live thyroid FNAs) to differentiate novice and expert participants was determined by an expert panel of otolaryngologists who frequently performed thyroid FNAs at Montefiore Medical Center.

Overall, 9 medical students, 7 novice residents/fellows, and 7 expert residents/fellows participated in this study. All participants received a standardized curriculum where they were taught the indications, complications, and techniques of ultrasound-guided thyroid FNA. Then, they were shown a demonstration of the simulation.

Using an ultrasound machine and a $25 \mathrm{G}$ needle, participants performed 6 trials of ultrasound-guided thyroid FNA (Fig. 2A,B) on the phantom. Each attempt was on a different nodule within the phantom, from the most medial to the most lateral nodules. Participants were allowed $3 \mathrm{~min}$ and unlimited attempts to identify and pass the needle through each nodule. Each trial was given a performance score from 0 to 100 (100 being maximum score). All scores began at 100 points and deductions were levied based on objective measurements: prolonged completion time, additional attempts, and errors (carotid injury, tracheal injury, transecting nodule, damaging model) (Table 1). This grading scale was devised with input from experienced attending otolaryngologists. Passage of the needle through the nodule was confirmed on ultrasound. Slide preparation and cytology were not simulated. 

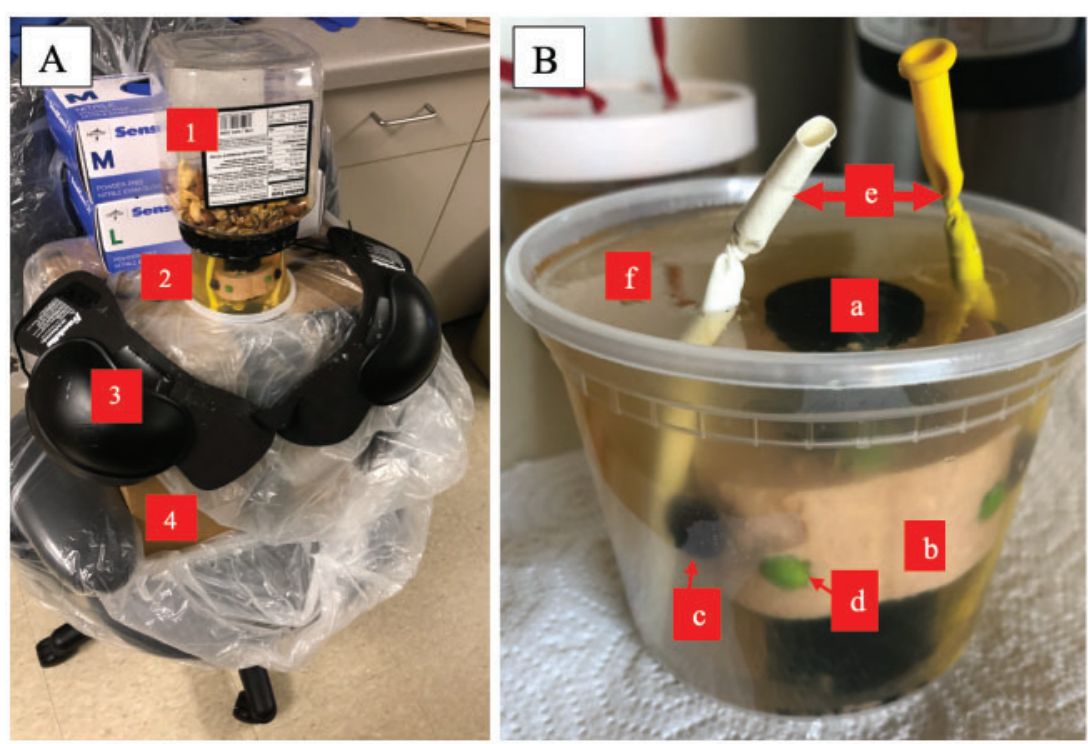

Figure 1. (A) The main components of the simulation phantom. 1, Container to represent the head; 2 , neck phantom (detailed in B); 3 , shoulder pads to simulate shoulders; 4 , box to simulate torso. (B) Components of the neck phantom: $a$, wire loom to simulate the trachea; $b$, Spam to simulate the thyroid; c, blueberries to simulate hyperechoic nodules; d, peas to simulate hypoechoic nodules; e, balloons filled with food coloring to simulate vessels; f, gelatin to simulate soft tissue of the neck.
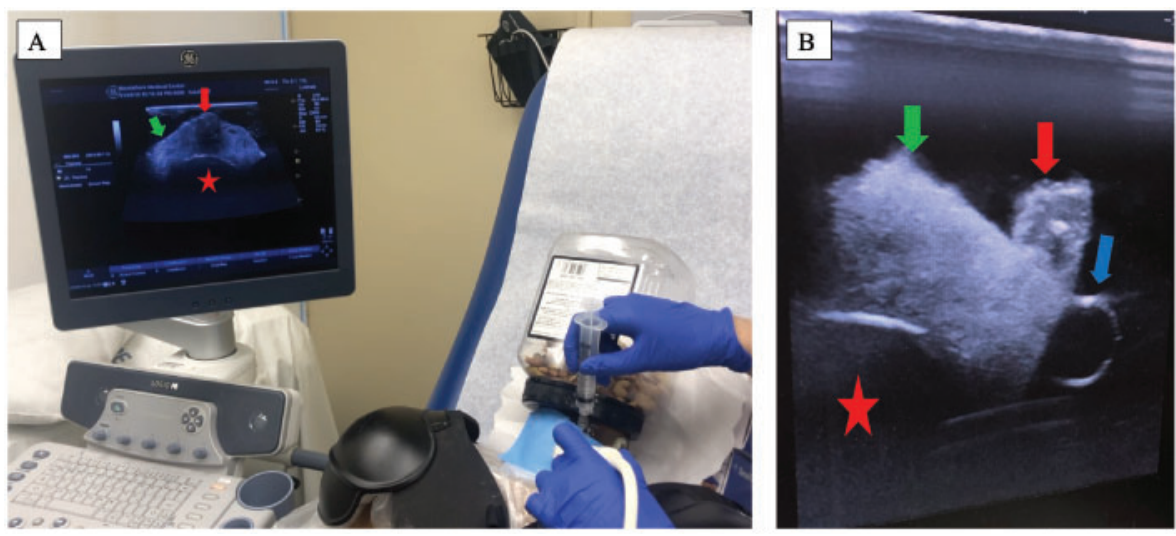

Figure 2. (A) Thyroid FNA phantom in use with ultrasound visualization. Trachea represented by a wire loom (red star), hypoechoic nodule represented by an embedded pea (red arrow), and thyroid represented by Spam (green arrow). (B) Ultrasound image of a lateral hyperechoic nodule represented by a blueberry (red arrow) adjacent to vessel represented by a balloon (blue arrow). Right hemisphere of the thyroid gland represented by Spam (green arrow). The trachea is represented by a wire loom (red star).

All participants answered the question "How beneficial is this phantom for practicing thyroid FNA" on a 5-point Likert scale. Expert residents/fellows participants also assessed "how realistic does ultrasound imaging feel" and "how realistic does needle haptic feedback feel" on a 5point Likert scale. Two attending endocrinologists who tested the phantom also answered the previous questions. These two endocrinologists did not participate in trials because their previous thyroid FNA experience was deemed to be too vast compared with other trial participants.

\section{Statistical analysis}

As scores for each group were not normally distributed and contained outliers, the Kruskal-Wallis $\mathrm{H}$ test (non-parametric counterpart to one-way ANOVA) was used to assess differences in scores between the three experiencelevel groups. Within each group, the related-samples Wilcoxon signed rank test was used to analyze the difference in score between the first and last trials (trial 1 versus trial 6) to assess improvement. Within each group, the nonparametric Friedman test was also used to measure progression of mean scores between nodules. All statistical analyses 
were performed using SPSS version 25.0 (IBM, Armonk, NY).

\section{Results}

Study participants were assigned to three groups depending on their lifetime experience with thyroid FNA. The medical student participants $(n=9)$ had no experience with performing thyroid FNA, the novices $(n=7)$ had an average of 0.7 thyroid FNA attempts (range, $0-2$ attempts) with 5 of 7 novices having zero FNA attempts, and experts $(n=7)$ had an average of 18.1 thyroid FNA attempts (range, 10-37 attempts). There were no drop outs, and every participant completed all six trials of the simulation.

The median scores on the simulation are displayed in Fig. 3. Performance between the three experience groups was proportionally related to experience as demonstrated by the significantly different mean rank scores at trials 1-6 $\left(\chi^{2}(2)=17.0, P<0.001\right)$. In trial 1 , the median score of

Table 1. Thyroid FNA simulation scoring for each nodule

\begin{tabular}{ll}
\hline Criteria & Points deducted \\
\hline Time & $\begin{array}{c}-0.33 \text { points for each } \\
\text { second }>30 \mathrm{~s}\end{array}$ \\
& $\begin{array}{c}20 \text { points for each } \\
\text { additional attempt }\end{array}$ \\
Accuracy & \\
Errors & -100 \\
Punctured carotid & -100 \\
Punctured trachea & -100 \\
Failure to biopsy after 3 min & -20 \\
Passing completely through nodule & -25 \\
Damaging model &
\end{tabular}

Scores start at 100 and points are deducted based on the criteria. experts (median, 100) was higher than that of novices (median, 33.3) and medical students (median, 0). This was reflected in pairwise comparisons using Dunn's procedure with Bonferroni correction: expert resident versus novice resident $(P=0.028)$, expert resident versus medical student $(P<0.001)$. In trial 1 , the pairwise comparison between medical students and novice residents showed no significant difference $(P=0.534)$. In all subsequent trials, the significant pairwise comparisons between expert residents and medical students persisted. Starting from trial 4, pairwise comparisons showed that novice residents performed significantly better than medical students.

The progression of median scores within each of the three experience-level groups is shown in Fig. 4. Between trial 1 and trial 6 , significant improvements in score were observed on Wilcoxon signed rank tests in both medical students $(P$ $=0.018)$ and novice residents $(P=0.018)$, but not in expert residents $(P=0.500)$. Differences between trials were significant in medical students $\left(\chi^{2}(5)=20.991, P=0.001\right)$ and novice residents $\left(\chi^{2}(5)=14.487, P=0.013\right)$, but not in expert residents $\left(\chi^{2}(5)=9.045, P=0.107\right)$. The only pairwise comparisons that were significant were between trials 1 and trial 5 in medical students $(P=0.030)$ and between trial 1 and trial 6 in novice residents $(P=0.032)$.

Feedback obtained is displayed in Fig. 5. Two endocrinology attendings tested the thyroid FNA simulation phantom. Both attendings strongly agreed or agreed that the phantom was beneficial for learning thyroid FNA and that the appearance on ultrasound was realistic. One attending agreed that haptic feel of needle penetration felt realistic, whereas the other felt neutral about it. All 7 expert residents participating in the study agreed or strongly agreed that the phantom was beneficial for learning thyroid FNA, 6 of 7 agreed or strongly agreed that the ultrasound appearance of the phantom was realistic, and 5 of 7 agreed that the haptic feel of the phantom was

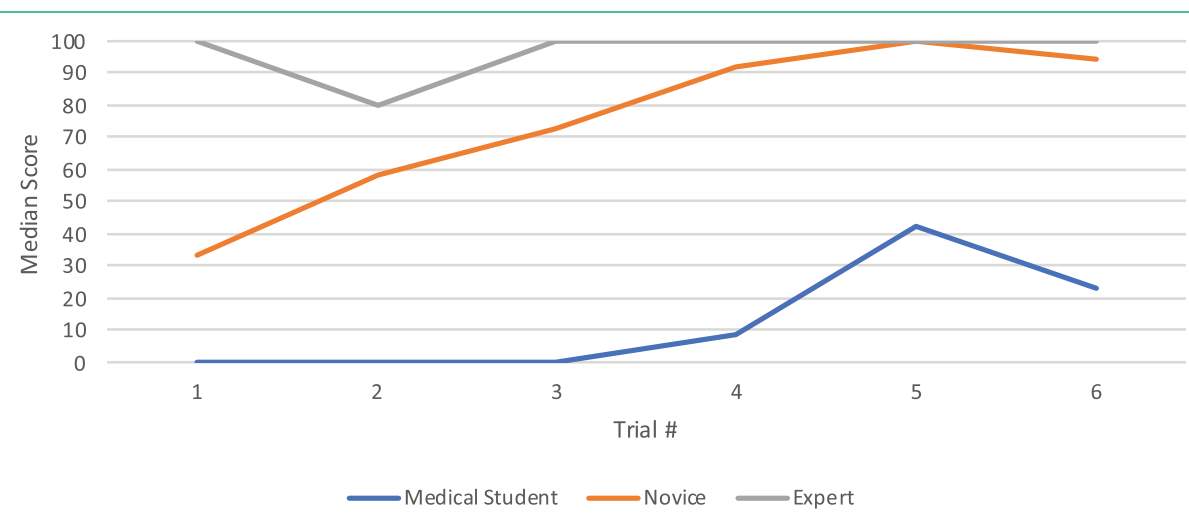

Figure 3. Progression of median scores for medical students $(n=9)$, novices $(n=7)$, and experts $(n=7)$. 


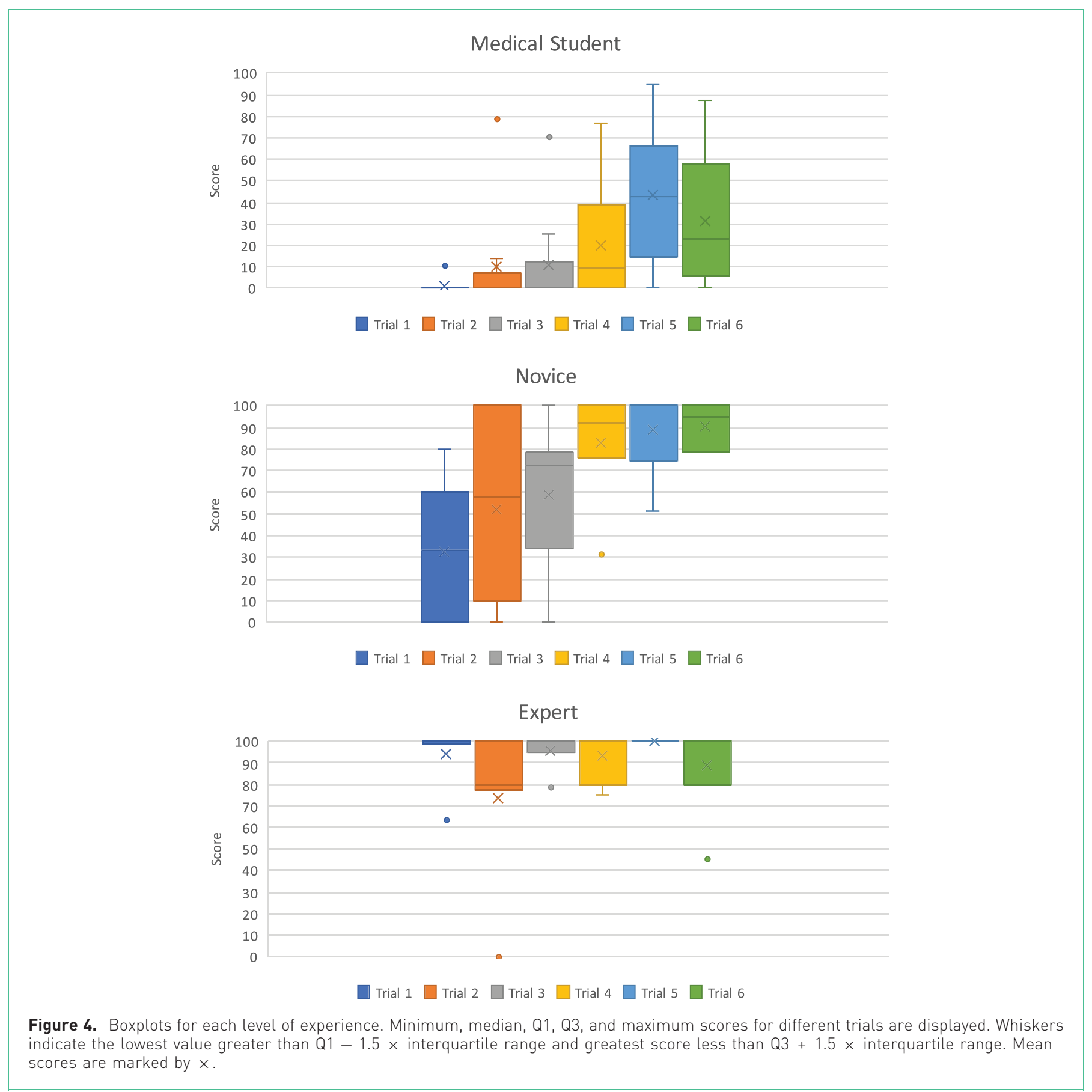

realistic. All 7 novice residents and 9 medical students strongly agreed or agreed that the phantom was beneficial for learning thyroid FNA. The most common positive feedback received was that the ultrasound appearance of the thyroid nodules was realistic. The most common negative feedback was that the slipperiness and softness of the gelatin would sometimes allow the needle to translate or glide.

The total cost of building each new model was US\$3. The phantom also requires about $20 \mathrm{~min}$ of assembly time and the gelatin to set overnight. Each model could be reused multiple times and stored in the refrigerator for over a month. As described in other FNA phantoms, track marks could be eliminated by placing the model in a microwave for 10-s intervals and solidifying the model in the refrigerator. ${ }^{6}$ Since the consistency and texture of the gelatin could change over time, phantoms used by study participants were less than 2 weeks old, and they were replaced when damaged or altered. This ensured consistent simulation conditions for study participants. Phantoms were microwaved 


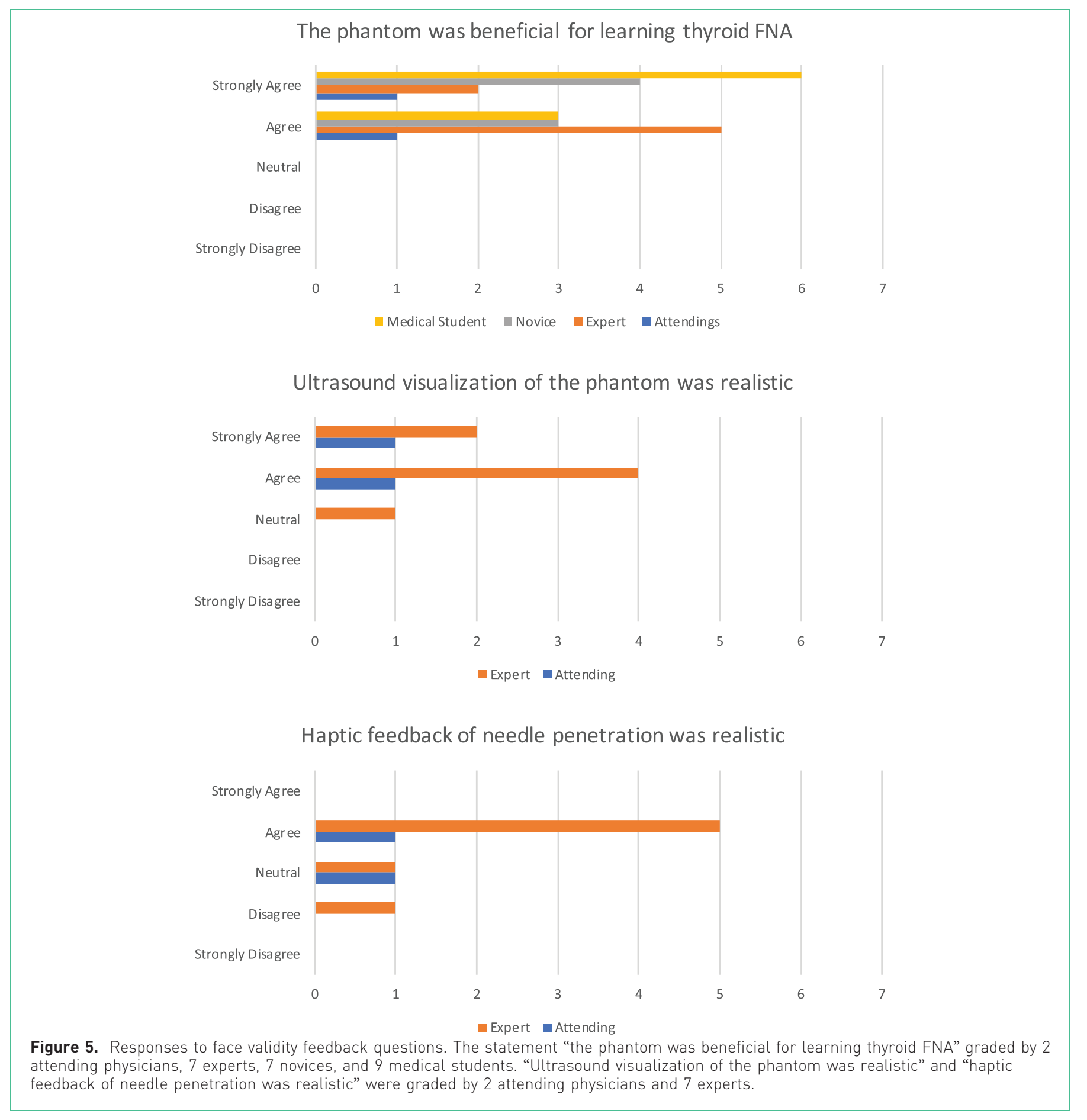

to remove track marks only a few times because new models were generated frequently.

\section{Discussion}

Our study demonstrates that an inexpensive ultrasoundguided realistic thyroid FNA phantom can be fabricated with accessible materials. Almost all of the materials used to create the thyroid FNA phantom can be found in a household or hospital, and the remainder can be readily purchased at a supermarket or online. The cost of the entire phantom was US $\$ 3$, which is comparable with a previously described superconcentrated gelatin phantom for thyroid FNA (US\$2-3) ${ }^{4}$ and cheaper than high-fidelity models such as the Blue Phantom (US\$1454.94) and a high-fidelity model constructed with a 3D mold (US\$1660). ${ }^{3}$ Similar to other low-cost ultrasound phantoms proposed in the literature, ${ }^{4,6,10,12-14}$ we used gelatin as our 
primary medium. In contrast to other thyroid FNA phantoms, ${ }^{6}$ our phantom replicates neck anatomy with good fidelity without using raw meat. The phantom also simulates the curvature of the neck and bulk properties of the head and torso, which can affect the angle of approach and ergonomics of the procedure. We believe that our phantom serves as a valuable educational tool for thyroid FNA training that can supplement existing well-respected thyroid FNA courses and references. ${ }^{15-18}$

To our knowledge, this is the first study to demonstrate both face and construct validity of a thyroid FNA phantom. The face validity of our low-cost, high-fidelity model was subjectively established through expert feedback, and discriminative construct validity was established by showing the phantom can distinguish between three levels of experience in performing thyroid FNA. Davis et al. ${ }^{19}$ studied 12 general surgery residents who had practiced thyroid FNA using a Blue Phantom Neck Mold with embedded olives and found that these residents had increased confidence levels, increased scores on a thyroid FNA written exam scores, and were able to achieve a diagnostic thyroid FNA on an actual patient. Zhang et al. ${ }^{8}$ proposed a tofu-based thyroid FNA phantom and found that puncture success rates increased after phantom use. Neither study formally validated the face and construct validity of the respective phantom.

Similar gelatin-based ultrasound-guided needle procedural trainers have been found in other disciplines. A ballistic gelatin ultrasound phantom for simulating glenohumeral intraarticular injections was found to increase learner comfort in medical students and residents. ${ }^{20}$ Use of gelatinbased spine phantoms have also been found to improve performance in ultrasound-guided cervical nerve blocks, ${ }^{21}$ lumbosacral spine facet joint injections, and medial branch nerve blocks. ${ }^{22}$ These models also involved immersing materials into a gelatin mold, but trained needle injection rather than aspiration. Gelatin phantoms have also been designed to simulate ultrasound-guided vascular access. ${ }^{14,23}$ Similar to our thyroid FNA phantom where balloons were filled with food coloring, vascular access phantoms were simulated vessels made of rubber tubing filled with food coloring.

\section{Face validity}

All participants at the three experience levels agreed that the thyroid FNA phantom was beneficial for learning the technique (Fig. 5). This serves as subjective evidence that the thyroid FNA phantom was a valuable tool for learning the technique across all levels of experience. Although the $1-\mathrm{cm}$ nodules incorporated in this phantom are smaller than those removed on biopsy in real life, we believe that training on more difficult nodules will allow development of better precision and will ultimately improve the diagnostic accuracy of more difficult nodules. Because the gelatin medium is transparent, uncovering the latex wrap "skin" of the phantom allowed users to correlate ultrasound imaging to true three-dimensional appearance. This is especially useful to help users understand how the needle, ultrasound probe, and the nodule are spatially related during thyroid FNA, a significant issue for novice users.

Besides one expert participant who was neutral, all experts and attending physicians agreed that the ultrasound appearance of the phantom was realistic (Fig. 5). This was reflected in many of the open-ended feedback responses for positive attributes of the thyroid FNA phantom. The selected materials had realistic echogenic properties that allowed visualization of relevant neck structures (trachea, thyroid, vessels) on ultrasound. The Spam could also be easily shaped into a thyroid and embedded with blueberries and peas to simulate nodules with variable echogenicity. Our method of building a mold and filling it with gelatin allowed for proper anatomic relationships to be consistently established.

Two of 7 expert residents and 1 of 2 attending physicians did not agree that the haptic feedback felt realistic (Fig. 5), and a commonly reported negative attribute of the phantom was that the gelatin medium was too soft and allowed for needle gliding. The gelatin concentration used in this phantom was $12 \%$, but could be adjusted up to $15 \%$ as reported in the literature ${ }^{4}$ to improve the firmness of the gelatin. Although some users did not feel that gelatin provided optimal haptic feedback, they did report that the haptic feedback of passing the needle through a blueberry nodule felt realistic.

\section{Discriminative construct validity}

To demonstrate discriminative construct validity, participants were assigned to different skill level groups based on their survey-reported number of thyroid FNA performed on live patients because we assumed proficiency increased with repetition. Novice residents with no experience performing thyroid FNA were assigned to a different group than medical students with no experience performing thyroid FNA because residents had greater knowledge and practical skills. Over the course of their medical training, residents would have acquired more experience with performing procedures in the neck (e.g. thyroidectomy), anatomic knowledge, and familiarity with ultrasound and needle manipulation than medical students.

Our results support the utility of our thyroid FNA phantom as a tool to evaluate skill level because performance scores in trial 1 were able to discriminate expert participants from 
novice participants and medical students (Figs 3 and 4). Although the median score of novice participants was higher than for medical students, this difference was not statistically significant and may be attributed to the fact that novice participants only had an average of 0.7 FNA attempts in their lifetime (versus $0 \mathrm{FNA}$ attempts in medical students). By trial 2, the significant differences between performance of scores in experts and novices began to disappear as the median scores of novice participants approximated those of expert participants. Novice participants also exhibited a significant improvement in scores between the first and last trials. These findings suggest that novice participant scores improve with repeated use of the phantom, and support use of the phantom in residency programs to develop proficiency in thyroid FNA.

Although medical student scores also improved significantly, a significant score difference between medical students and experts persisted through all trials. By trial 4, a significant score difference between novices and medical students appeared and novice participants exhibited earlier and larger improvements in median scores than medical students (Figs 3 and 4). These findings suggest that novice participants may have derived more benefit from the thyroid FNA because of their increased anatomic knowledge and practical skillset compared with medical students. To overcome this disadvantage, medical students may require more than 6 repetitions using the phantom before being able to experience the same benefit as novice participants.

Expert participants did not yield significant improvement through repeated use of the phantom likely because their median baseline score was already at 100 . As the location and size of the nodules and thyroid are customizable, it is possible to decrease nodule size and increase nodule depth to help experts refine their skills with more difficult nodules. It is also possible that a more stringent grading criteria is necessary to observe improvement in performance.

\section{Limitations and next steps}

As with other simulation studies, sample size was a limitation due to the otolaryngology and endocrinology fellowship programs being relatively small. The size of our study sample is comparable, but slightly smaller, than other similar simulation validation studies. Moreover, as there was a lack of a validated thyroid FNA scoring tool, we devised an original scoring scale. Although not validated, this scale was designed with the input of expert otolaryngologists and was based on objective criteria. The phantom also requires about $20 \mathrm{~min}$ of assembly time and the gelatin to set overnight, which requires some planning in advance. However, phantoms can be reused, stored for $>1$ month, and be produced more efficiently if multiple phantoms are fabricated simultaneously.
The next step of this study is to establish predictive validity by first randomizing novice residents to receive simulation training or to a control group and them comparing their performance scores of thyroid FNA performed on cadavers and live patients. This would establish that skills developed using the thyroid FNA phantom translate to improved performance of the procedure in vivo.

\section{Conclusion}

We designed a low-cost thyroid FNA phantom with good fidelity that can be constructed using easily accessible materials. Face and discriminate construct validity were established by having participants of various skill levels test the phantom and provide feedback. The proposed thyroid FNA phantom can be used to train procedural skills in a residency program and evaluate skill acquisition.

\section{Acknowledgements}

The authors would like to acknowledge the medical students, residents, fellows, and attending physicians who participated in the study.

\section{Conflict of interest}

No competing financial interests exist.

\section{References}

1. American Thyroid Association (ATA) Guidelines Taskforce on Thyroid Nodules and Differentiated Thyroid Cancer; Cooper DS, Doherty GM, Haugen BR, Kloos RT, Lee SL, et al. Revised American Thyroid Association management guidelines for patients with thyroid nodules and differentiated thyroid cancer. Thyroid 2009; 19: 1167-1214. https://doi. org/10.1089/thy.2009.0110.

2. Jandu $\mathrm{M}$, Webster $\mathrm{K}$. The role of operator experience in fine needle aspiration cytology of head and neck masses. Int J Oral Maxillofac Surg 1999; 28: 441-444. https://doi.org/10. 1016/S0901-5027(99)80057-5.

3. Baba $M$, Matsumoto $K$, Yamasaki $N$, Shindo $H$, Yano $H$, Matsumoto $\mathrm{M}$, et al. Development of a tailored thyroid gland phantom for fine-needle aspiration cytology by threedimensional printing. J Surg Educ 2017; 74: 1039-1046. https://doi.org/10.1016/j.jsurg.2017.05.012.

4. Abraham D. A method using superconcentrated gelatin and a novel phantom suspension system for ultrasound-guided thyroid biopsy training. Thyroid 2014; 24: 1662-1663. https://doi. org/10.1089/thy.2014.0057. 
5. Nolting L, Hunt P, Cook T, Douglas B. An inexpensive and easy ultrasound phantom: a novel use for SPAM. J Ultrasound Med 2016; 35: 819-822. https://doi.org/10.7863/ultra.14.06023.

6. Richardson C, Bernard S, Dinh VA. A Cost-effective, gelatinbased phantom model for learning ultrasound-guided fineneedle aspiration procedures of the head and neck. J Ultrasound Med 2015; 34: 1479-1484. https://doi.org/10. 7863/ultra.34.8.1479.

7. Shidham VB, Varsegi GM, D'Amore K, Shidham A. Preparation and using phantom lesions to practice fineneedle aspiration biopsies. J Vis Exp. 2009; (31): 1404. https://doi.org/10.3791/1404.

8. Zhang YF, Li H, Wang XM. Technical report: a cost-effective, easily available tofu model for training residents in ultrasound-guided fine needle thyroid nodule targeting punctures. Korean J Radiol 2019; 20: 166-170. https://doi.org/10.3348/kjr. 2017.0772.

9. Vieira SL, Pavan TZ, Junior JE, Carneiro AA. Paraffin-gel tissue-mimicking material for ultrasound-guided needle biopsy phantom. Ultrasound Med Biol 2013; 39: 2477-2484. https://doi.org/10.1016/j.ultrasmedbio.2013.06.008.

10. Sevak S, Lurvey B, Woodfin AA, Hothem Z, Callahan RE, Robbins J, et al. solid, cystic, and tubular: novice ultrasound skills training using a versatile, affordable practice model. J Surg Educ 2018; 75: 1403-1409. https://doi.org/10.1016/j. jsurg.2018.02.010.

11. Schwartz CM, Ivancic RJ, McDermott SM, Bahner DP. Designing a low-cost thyroid ultrasound phantom for medical student education. Ultrasound Med Biol 2020; 46: 1545-1550. https://doi.org/10.1016/j.ultrasmedbio.2020.01.033.

12. Chao SL, Chen KC, Lin LW, Wang TL, Chong CF. Ultrasound phantoms made of gelatin covered with hydrocolloid skin dressing. J Emerg Med 2013; 45: 240-243. https://doi.org/10.1016/j.jemermed.2012.11.022.

13. McNamara MP Jr, McNamara ME. Preparation of a homemade ultrasound biopsy phantom. J Clin Ultrasound 1989; 17: 456-458. https://doi.org/10.1002/jcu.1870170617.

14. Morrow DS, Broder J. Cost-effective, reusable, leak-resistant ultrasound-guided vascular access trainer. J Emerg Med 2015; 49: 313-317. https://doi.org/10.1016/j.jemermed.2015.04.005.
15. College of American Pathologists. Ultrasound-Guided FineNeedle Aspiration Advanced Practical Pathology Program. Available from: https://learn.cap.org/activity/3371965/detail. aspx (accessed 11 June 2020).

16. American Association of Clinical Endocrinologists. The Endocrine Certification in Neck Ultrasound Program. Available from: https://www.aace.com/education/certificationneck-ultrasound (accessed 11 June 2020).

17. Rollins SD. Teaching FNA techniques and ultrasound guided FNA. Cancer Cytopathol 2019; 127: 7-8. https://doi.org/10. 1002/cncy.22064.

18. Fish S, Gianoukakis AG. Thyroid Ultrasound Course Presented at the 81st Annual Meeting of the American Thyroid Association. Available from: http://www.thyroid.org/professionals/trainees-corner/trainee-education/7th-annual-atafellows-track-program/ (accessed 11 June 2020).

19. Davis JR, Hale AL, Ewing JA, Lokey JS. Instituting ultrasound-guided FNA for thyroid nodules into a general surgery residency program: what we learned. J Surg Educ 2018; 75: 594-600. https://doi.org/10.1016/j.jsurg.2017.09.031.

20. Shah A. A Low-cost, reusable, ballistic gelatin ultrasound phantom for simulation of glenohumeral intraarticular injections. AEM Educ Train 2018; 2: 169-173. https://doi.org/10. 1002/aet2.10081.

21. Kwon SY, Kim JW, Cho MJ, Al-Sinan AH, Han YJ, Kim YH. The efficacy of cervical spine phantoms for improving resident proficiency in performing ultrasound-guided cervical medial branch block: a prospective, randomized, comparative study. Medicine (Baltimore) 2018; 97: e13765. https://doi.org/10. 1097/MD.0000000000013765.

22. Kwon SY, Hong SH, Kim ES, Park HJ, You Y, Kim YH. The efficacy of lumbosacral spine phantom to improve resident proficiency in performing ultrasound-guided spinal procedure. Pain Med 2015; 16: 2284-2291. https://doi.org/10.1111/pme. 12870.

23. Kocharyan H, Kallini J, Aida SK, Harvill M. A cost-effective alternative formulation of ultrasound phantom for vascular access instruction: cost-effective hands-on procedural training. J Vasc Access 2018; 19: 328-329. https://doi.org/10. $1177 / 1129729817747533$. 